\title{
A NOTE ON THE DEPENDENCE STRUCTURE OF THE TWO-STATE MARKOVIAN ARRIVAL PROCESS
}

\author{
PEPA RAMÍREZ-COBO* AND \\ EMILIO CARRIZOSA, ${ }^{* *}$ University of Seville
}

\begin{abstract}
The Markovian arrival process generalizes the Poisson process by allowing for dependent and nonexponential interarrival times. We study the autocorrelation function of the twostate Markovian arrival process. Our findings show that the correlation structure of such a process has a very specific pattern, namely, it always converges geometrically to zero. Moreover, the signs of the autocorrelation coefficients are either constant or alternating.

Keywords: Markovian arrival process; Markov renewal process; autocorrelation function 2010 Mathematics Subject Classification: Primary 60G55
\end{abstract}

Secondary $60 \mathrm{~J} 05$

\section{Introduction}

The Markovian arrival process (MAP) was introduced by Neuts [5] as a wide class of versatile point processes that generalize the Poisson arrival process by allowing for dependent and nonexponential interarrival times. The MAP, which includes as special cases the wellknown phase-type renewal and Markov-modulated Poisson processes, has been considered as a model in various contexts where dependent data are observed. In particular, it is well known that teletraffic arrivals are not well modeled by a Poisson process and various works have used the MAP as an alternative in this context. For examples, see [2] and [7]. Also, in reliability theory, where correlated traces are usually found in practice, the MAP has been suggested for modeling the interfailure time or the arrivals of shocks that cause the failure of a system; see, for instance, [4]. From a queueing theory perspective, where the usual assumption of independent interarrival times may be restrictive, the MAP has been proven to be a useful process. Its capability to model dependent observations makes the MAP an appealing model for governing the arrival process of a queueing system and, as a consequence, numerous works dealing with theoretical properties of the MAP/G/1 queueing system can be found in the literature [3], [6]. The autocorrelation function of a sequence of interarrival times of a MAP has been known in closed form since 1979; see [1] and [5]. However, it is of interest to study which types of dependence structures may be well modeled with a MAP, and to the best of the authors' knowledge, no analysis concerning the behavior of the autocorrelation function has been undertaken before. Since the MAPs are over-parametrized processes, usually two states, at most three, are enough to capture the data behavior, and indeed in most applications, the two-state case has been considered; see, for example, [7], [9], or [10]. In this note we study the

Received 2 November 2010; revision received 4 October 2011.

* Postal address: Institute of Mathematics, University of Seville, Avda. Reina Mercedes s/n, 41012 Seville, Spain.

Email address: jrcobo@us.es

** Postal address: Department of Statistics and Operations Research, University of Seville, Avda. Reina Mercedes s/n, 41012 Seville, Spain. Email address: ecarrizosa@us.es 
two-state MAP, referred to hereafter as the $\mathrm{MAP}_{2}$. We prove that the autocorrelation function of the $\mathrm{MAP}_{2}$ has a particular form, namely, it decreases geometrically to zero as a function of the time lags. This shows in particular that the $\mathrm{MAP}_{2}$ may not be adequate to model seasonal data.

\section{The MAP}

A number of works have described the MAP in detail; see, for example, [1] and [3]. With regards to the $\mathrm{MAP}_{2}$, we refer the reader to the descriptions given in [7] and [8]. A stationary $\mathrm{MAP}_{2}$ is represented by $\left\{\lambda, P_{0}, P_{1}\right\}$, where $\lambda=\left(\lambda_{1}, \lambda_{2}\right)$ denotes the exponential rates, and

$$
P_{0}=\left(\begin{array}{ll}
0 & x \\
z & 0
\end{array}\right) \quad \text { and } \quad P_{1}=\left(\begin{array}{cc}
y & 1-x-y \\
w & 1-z-w
\end{array}\right)
$$

represent the transition probabilities, where $x=p_{120}, y=p_{111}, z=p_{210}$, and $w=p_{212}$. The stationary $\mathrm{MAP}_{2}$ behaves as follows: the initial state $i_{0} \in\{1,2\}$ is generated according to the stationary probability vector $\pi=(\pi, 1-\pi)$ and at the end of an exponentially distributed sojourn time in state $i$, with mean $1 / \lambda_{i}$, two possible state transitions can occur. Firstly, with probability $0 \leq p_{i j 1} \leq 1$, a single arrival occurs and the $\operatorname{MAP}_{2}$ enters a state $j \in\{1,2\}$, which may be the same $(j=i)$ as or different $(j \neq i)$ to the previous state. Secondly, with probability $0 \leq p_{i j 0} \leq 1$, no arrival occurs and the $\mathrm{MAP}_{2}$ enters a different $(j \neq i)$ state. Without loss of generality, we assume that $\lambda_{1} \geq \lambda_{2}>0$. The $\mathrm{MAP}_{2}$ can also be characterized in terms of the rate matrices, $D_{0}=\left(d_{i j 0}\right)_{i, j \in\{1,2\}}$ and $D_{1}=\left(d_{i j 1}\right)_{i, j \in\{1,2\}}$, where $d_{i i 0}=-\lambda_{i}, d_{i j 0}=\lambda_{i} p_{i j 0}$ for $i \neq j$, and $d_{i j 1}=\lambda_{i} p_{i j 1}$ for $i, j \in\{1,2\}$. The matrix $D_{0}$ is assumed to be stable, which implies that it is nonsingular and the sojourn times are finite with probability 1 (which in turn implies that the arrival process does not terminate). The definitions of $D_{0}$ and $D_{1}$ implies that $D=D_{0}+D_{1}$ is the infinitesimal generator of the underlying Markov process, with stationary probability vector $\boldsymbol{\pi}$, computed as $\boldsymbol{\pi} D=\mathbf{0}$.

Some properties of the $\mathrm{MAP}_{2}$ (and of general $m$-state MAPs), found for example in [1], are as follows. Firstly, it is known that the MAP can be regarded as a Markov renewal process. If $X_{n}$ denotes the state of the MAP at the time of the $n$th arrival, and $T_{n}$ the time between the $(n-1)$ th and $n$th arrival, then $\left\{X_{n-1}, T_{n}\right\}_{n=1}^{\infty}$ is a Markov renewal process, and, in particular, $\left\{X_{n}\right\}_{n=1}^{\infty}$ is a Markov chain whose transition matrix is easily derived as

$$
P^{\star}=\left(I-P_{0}\right)^{-1} P_{1} \text {. }
$$

Let $T$ denote the time between two successive arrivals in the stationary version of a MAP. Then, the cumulative distribution function of $T$ is

$$
F_{T}(t)=\left(\pi D_{1} \boldsymbol{e}\right)^{-1} \pi D_{1}\left(I-\mathrm{e}^{D_{0} t}\right)\left(-D_{0}\right)^{-1} D_{1} \boldsymbol{e} \text { for } t \geq 0,
$$

where $\boldsymbol{e}$ is a unit column vector of the same order as the MAP. Alternatively, since $\left(D_{0}+D_{1}\right) \boldsymbol{e}=$ 0, (2.2) can be rewritten as

$$
F_{T}(t)=1-\phi \mathrm{e}^{D_{0} t} \boldsymbol{e} \text { for } t \geq 0,
$$

and, therefore, $T$ follows a phase-type (PH) distribution with representation $\left(\phi, D_{0}\right)$, where $\boldsymbol{\phi}=\left(\boldsymbol{\pi} D_{1} \boldsymbol{e}\right)^{-1} \pi D_{1}$ is the stationary probability distribution satisfying $\boldsymbol{\phi} P^{\star}=\boldsymbol{\phi}$ (see [8]). The mean and variance of $T$ are given by

$$
\begin{gathered}
\mu=\frac{\pi D_{1}\left(-D_{0}\right)^{-1} \boldsymbol{e}}{\boldsymbol{\pi} D_{1} \boldsymbol{e}}=\frac{1}{\boldsymbol{\pi} D_{1} \boldsymbol{e}}, \\
\sigma^{2}=2 \mu \boldsymbol{\pi}\left(-D_{0}\right)^{-1} \boldsymbol{e}-\mu^{2} .
\end{gathered}
$$


Finally, the autocorrelation function of the interarrival times $\left\{T_{n}\right\}_{n=1}^{\infty}$ in the stationary version of the $\mathrm{MAP}_{2}$, the function we focus on in this work, is given by

$$
\rho(k)=\rho\left(T_{1}, T_{k+1}\right)= \begin{cases}\frac{\mu\left(\pi\left[\left(-D_{0}\right)^{-1} D_{1}\right]^{k}\left(-D_{0}\right)^{-1} \boldsymbol{e}-\mu\right)}{\sigma^{2}}, & k>0, \\ 1, & k=0,\end{cases}
$$

where $k$ represents the time lag.

\section{Main result}

It can be seen from (2.1) that any $\mathrm{MAP}_{2}$ in its stationary version is characterized by six parameters: the exponential rates $\lambda=\left(\lambda_{1}, \lambda_{2}\right)$ and the transition probabilities (or, alternatively, transition rates) $\{x, y, z, w\}$. However, to study the autocorrelation function (2.6), for ease of notation, there is no loss of generality in assuming the alternative parametrization in terms of five parameters:

$$
\tilde{\lambda}=(1, u), \quad \tilde{D}_{0}=\left(\begin{array}{cc}
-1 & x \\
u z & -u
\end{array}\right), \quad \tilde{D}_{1}=\left(\begin{array}{cc}
y & 1-x-y \\
u w & u(1-z-w)
\end{array}\right) .
$$

Note that representation (3.1) is derived from the definitions of $D_{0}$ and $D_{1}$ by dividing each component by $\lambda_{1}$, so that $u=\lambda_{2} / \lambda_{1}$. It is immediate that $\tilde{\boldsymbol{\pi}}=\pi, \tilde{\mu}=\lambda_{1} \mu$, and $\tilde{\sigma}^{2}=\lambda_{1}^{2} \sigma^{2}$; therefore, it follows easily that the autocorrelation function $\rho(k),(2.6)$, derived from the original representation is the same as that derived from representation (3.1), $\tilde{\rho}(k)$.

It is tedious but straightforward to prove that, since $\lambda_{1} \geq \lambda_{2}>0, D_{0}$ is stable, both the mean $\mu$ and variance $\sigma^{2}$ are strictly positive and finite, and, finally, the steady-state probability $\pi$ exists; then we obtain the following constraints on the parameters:

$$
0<u \leq 1, \quad(x, z) \neq(1,1), \quad(y, z, w) \neq(1,0,0) .
$$

In what follows we assume that (3.2) is satisfied.

Next, we present the major contribution of this work.

Theorem 3.1. Let a MAP $P_{2}$ be characterized by $\{x, y, w, z, u\}$ as in (3.1), and let $\rho(k)$ denote the corresponding autocorrelation function. Then, $\rho(k)$ has the form $p \cdot q^{k}$ for some $p, q,|q|<1$. In particular,

$$
|\rho(k)| \geq|\rho(k+1)| \text { for all } k>0 \text { and } \lim _{k \rightarrow 0} \rho(k)=0 .
$$

Proof. We begin by pointing out that the variance $\sigma^{2}$ given in (2.5) can be written as

$$
\sigma^{2}=\mu \tau
$$

where

$$
\tau=2 \pi\left(-D_{0}\right)^{-1} \boldsymbol{e}-\mu .
$$

In terms of the model parameters $\{x, y, z, w, u\}, \tau$ is found to be given by

$$
\tau=\frac{\tau_{0}(x, y, z, w, u)}{u(1-y+u z+u w)(z x-1)(-y z-w+z x+w x-1+y)},
$$

where

$$
\tau_{0}=\alpha(x, y, z, w) u^{2}+\beta(x, y, z, w) u+\gamma(x, y, z, w)
$$


and

$$
\begin{aligned}
& \alpha(x, y, z, w)=(z+w)\left(x z^{2}+[(w-2) x+2 y-1] z-2 w x+2-2 y+w\right), \\
& \beta(x, y, z, w)=-2(z+w)^{2}+2(z+w)(y z+1+w-y) x-2(z-1)(y-1)(y z+w), \\
& \gamma(x, y, z, w)=(y-1)(-2 y z+y+y z x-2 w-1+2 w x+z x) .
\end{aligned}
$$

By (3.4) and since $\mu>0$ is assumed, the autocorrelation function $\rho(k)$ as in (2.6) becomes

$$
\rho(k)=\frac{\pi\left[\left(-D_{0}\right)^{-1} D_{1}\right]^{k}\left(-D_{0}\right)^{-1} \boldsymbol{e}-\mu}{\tau}
$$

for $k>0$. Consider the numerator in (3.6). It can be easily seen that $\left(-D_{0}\right)^{-1} D_{1}$ has spectral decomposition

$$
\left(-D_{0}\right)^{-1} D_{1}=Q \Delta Q^{-1}
$$

where

$$
Q=\left(\begin{array}{cc}
1 & \frac{-1+y+x w+x z}{y z+w} \\
1 & 1
\end{array}\right), \quad \Delta=\left(\begin{array}{cc}
1 & 0 \\
0 & \frac{-(x w-w-y z+y)}{z x-1}
\end{array}\right) .
$$

Therefore,

$$
\left[\left(-D_{0}\right)^{-1} D_{1}\right]^{k}=Q \Delta^{k} Q^{-1},
$$

and, hence, taking into account (3.5), it is easy to check that (3.6) becomes

$$
\rho(k)=\frac{(-1)^{k} \kappa(x w-w-y z+y)^{k}(u z-u-x+1)}{\tau_{0}(z x-1)^{k}},
$$

where $\kappa$ is given by

$$
\kappa=\left(x u z^{2}+2 w x u z+y u z-u z+y u w+x u w^{2}-w u-y^{2} z+y z-w y+w\right) .
$$

By (3.7),

$$
\rho(k)=p \cdot q^{k}
$$

where

$$
p=\frac{\kappa(u z-u-x+1)}{\tau_{0}} \text { and } q=\frac{x w-w-y z+y}{1-z x} .
$$

In order to show that $\left\{a_{k}\right\}_{k>0}=\{|\rho(k)|\}_{k>0}$ is a nonincreasing sequence, it is sufficient to prove that $|x w-w-y z+y| \leq 1-z x$. Since $0 \leq x, y, z, w \leq 1, x+y \leq 1$, and $z+w \leq 1$, we have

$$
x w-w-y z+y \leq x(1-z)-0-0+y=(x+y)-x z \leq 1-x z
$$

and

$$
-x w+w+y z-y \leq-0+w+(1-x) z-0=(w+z)-x z \leq 1-x z .
$$

Combining (3.9) and (3.10), we have $|x w-w-y z+y| \leq 1-z x$, as asserted.

Moreover, we are in position to show that, when $|\rho(1)|>0$, the inequality above is strict, i.e. $|q|<1$. Indeed, if $|q|=1$, all the inequalities in either (3.9) or (3.10) are equalities. In the former case, it would follow that $x w=x(1-z), w=0, y z=0$, and $x+y=1$. 
This happens only when $(x, y, z, w)=(0,1,0,0)$ or $(x, y, z, w)=(1,0,1,0)$, but these two cases are forbidden by assumption (3.2). In the latter case, we would have $x w=0$, $y z=(1-x) z, y=0$, and $z+w=1$. But this happens only when $(x, y, z, w)=(1,0,1,0)$ or $(x, y, z, w)=(0,0,0,1)$. The first case is forbidden by (3.2), and in the second case we have $\rho(1)=0$. Hence, either $\rho(1)=0$, and, thus, $\rho(k)=0$ for all $k$, or $|q|<1$, and, thus, $|\rho(k+1)|<|\rho(k)|$ for all $k$.

Remark 3.1. By (3.8), it is clear that, when $\rho\left(k_{0}\right)=0$ for any $k_{0}>0$, then $\rho(k)=0$ for all $k>0$.

Remark 3.2. Expression (3.8) obtained for $\rho(k)$ implies that the signs of $\{\rho(k)\}_{k>0}$ are either constant or alternating in $k$. The following four patterns are, in principle, possible.

Pattern 1: $\rho(k) \geq 0$ for all $k>0$. This pattern occurs when $p, q \geq 0$. As an example, consider the $\mathrm{MAP}_{2}$ where

$$
\{u, x, y, z, w\}=\{0.1,0.05,0.95,0.1,0.9\} .
$$

It is easy to check that $p=0.3368, q=0.8592$, and the autocorrelation function is

$$
\begin{aligned}
& \rho(1)=0.2895, \quad \rho(2)=0.2137, \quad \rho(3)=0.1837, \quad \rho(4)=0.1578 \text {, } \\
& \rho(5)=0.1356, \quad \rho(6)=0.1165, \quad \rho(7)=0.1001, \quad \rho(8)=0.0862 \text {, }
\end{aligned}
$$

etc.

Pattern 2: $\rho(k) \leq 0$ for all $k>0$. This happens when $p \leq 0$ and $q \geq 0$. For example, consider the $\mathrm{MAP}_{2}$ where

$$
\{u, x, y, z, w\}=\{0.9396,0.2183,0.4785,0.5583,0.1051\} .
$$

Here, $p=-0.0133, q=0.1471$, and the autocorrelation function is given by

$$
\begin{array}{ll}
\rho(1)=-0.00195, & \rho(2)=-0.00028, \\
\rho(3)=-4 \times 10^{-5}, & \rho(4)=-6.3 \times 10^{-6},
\end{array}
$$

etc.

Pattern 3: $\rho(2 k) \geq 0$ and $\rho(2 k+1) \leq 0$ for all $k>0$. This pattern occurs when $p, q \leq 0$. As an illustration, consider the $\mathrm{MAP}_{2}$ where

$$
\{u, x, y, z, w\}=\{0.8233,0.2813,0.0092,0.5502,0.3589\} .
$$

Then, $p=0.3003, q=-0.0272$, and the autocorrelation function is

$$
\rho(1)=0.0081, \quad \rho(2)=-0.0024, \quad \rho(3)=0.0007, \quad \rho(4)=-0.0002,
$$

etc.

Pattern 4: $\rho(2 k) \leq 0$ and $\rho(2 k+1) \geq 0$ for all $k>0$. This is the case where $p \geq 0$ and $q \leq 0$. As an example, consider the $\mathrm{MAP}_{2}$ where

$$
\{u, x, y, z, w\}=\{0.0421,0.0055,0.1613,0.0473,0.8523\} .
$$


In this case, $p=0.3157, q=-0.6941$, and the autocorrelation function is

$$
\begin{array}{llll}
\rho(1)=-0.2191, & \rho(2)=0.1521, & \rho(3)=-0.1056, & \rho(4)=0.0733, \\
\rho(5)=-0.0509, & \rho(6)=0.0353, & \rho(7)=-0.0245, & \rho(8)=0.0170,
\end{array}
$$

etc.

After simulating one million realizations from $\mathrm{MAP}_{2} \mathrm{~s}$, we have empirical evidence that patterns 1 and 4 are more common in practice (around 65\% of the time) than patterns 2 and 3 . In addition, in all cases it was observed that when either pattern 2 or pattern 3 occurs, then $|\rho(1)|<0.1$, thus leading to close to zero correlation values.

\section{An example}

We exemplify our approach using a real data set. A sequence of 972 monthly rainfall data, corresponding to 81 consecutive years (1903-1983) and obtained from the Spanish National Climate Center (see http://www.aemet.es), has been analyzed. Specifically, the Bayesian algorithm in [7] to fit a $\mathrm{MAP}_{2}$ to a given data set was used. The method provides an estimation of the marginal PH distribution (2.3) and also of the autocorrelation function (2.6), as a measure of the joint behavior of the data. The monthly rainfall sample mean was $\mu=37.9059$ and the sample variance $\sigma^{2}=2234.9$; the estimated values through a $\mathrm{MAP}_{2}$ were 38.2678 and 2481, respectively. In Figure 1 we present the estimated (dotted line) and the empirical (solid line) cumulative distribution functions (CDFs) of the data. From this, it can be concluded that the estimated PH distribution fits the empirical CDF of the data properly. In Table 1 we give the empirical and predictive autocorrelation functions. It can be seen from the second column that the correlation structure of the data does not match that of a theoretical $\mathrm{MAP}_{2}$, described by Theorem 3.1. Therefore, the $\mathrm{MAP}_{2}$ fails in fitting the values of the data autocorrelation function, as the third column of Table 1 shows.

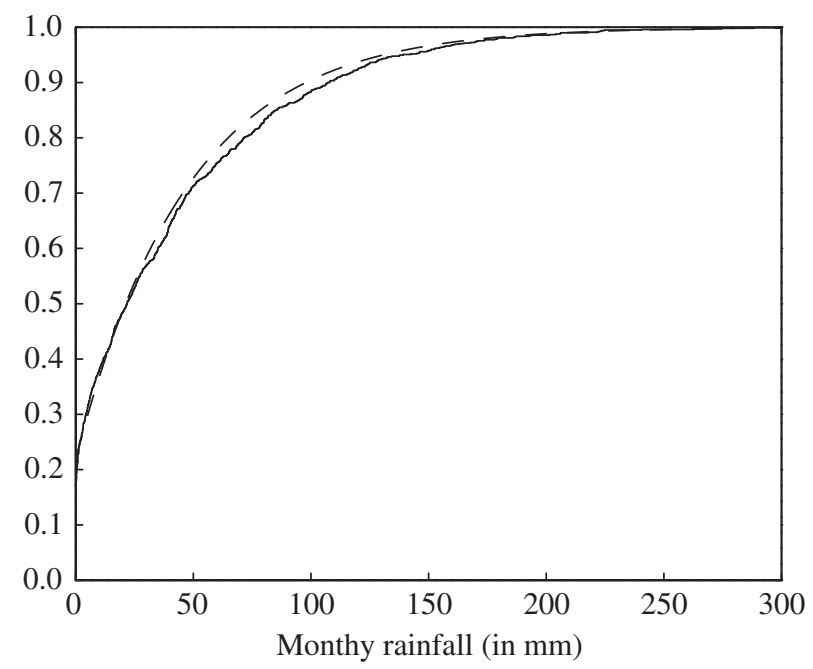

FIGURE 1: Empirical (solid line) and fitted by a $\mathrm{MAP}_{2}$ (dashed line) CDFs of monthly rainfall data. 
TABLE 1: Real and predictive autocorrelation functions.

\begin{tabular}{rcc}
\hline$k$ & Empirical $\rho(k)$ & Estimated $\rho(k)$ \\
\hline 1 & 0.2638 & 0.0592 \\
2 & 0.0997 & 0.0203 \\
3 & -0.0186 & 0.0071 \\
4 & -0.1169 & 0.0025 \\
5 & -0.2327 & 0.0009 \\
6 & -0.2217 & 0.0003 \\
7 & -0.2046 & 0.0001 \\
8 & -0.1431 & 0.0000 \\
9 & 0.0133 & 0.0000 \\
10 & 0.0977 & 0.0000 \\
11 & 0.2246 & 0.0000 \\
12 & 0.2886 & 0.0000 \\
\hline
\end{tabular}

Although the $\mathrm{MAP}_{2}$ is a potential model capable of fitting dependent observations, this example highlights that not all dependence patterns will be properly modeled by a MAP 2 . In particular, those data which do not satisfy (3.3) (presenting a seasonal or cyclical behavior for example) will not be well fitted with a $\mathrm{MAP}_{2}$. Experience has shown that it is worthwhile examining the data for evidence of a structure similar to that provided by Theorem 3.1, before considering the modeling by a $\mathrm{MAP}_{2}$.

It is natural to wonder if richer correlation patterns are obtained with higher-order MAPs, and, indeed, they are. For example, consider the $\mathrm{MAP}_{3}$ defined by

$$
P_{0}=\left(\begin{array}{ccc}
0 & 0.1368 & 0.0455 \\
0.1403 & 0 & 0.0093 \\
0.0306 & 0.0099 & 0
\end{array}\right), \quad P_{1}=\left(\begin{array}{ccc}
0.0243 & 0.5665 & 0.2269 \\
0.0026 & 0.0132 & 0.8346 \\
0.0471 & 0.0079 & 0.9045
\end{array}\right),
$$

with exponential rates given by $\lambda=(6.7527,7.9326,54.1947)$. The autocorrelation function (2.6) is

$$
\begin{gathered}
\rho(1)=0.1127, \quad \rho(2)=-0.0028, \quad \rho(3)=-0.0025, \\
\rho(4)=-6.074 \times 10^{-5}, \quad \rho(5)=5.151 \times 10^{-5},
\end{gathered}
$$

whose signs are neither constant nor alternating, against Remark 2. As another example, the $\mathrm{MAP}_{3}$ given by

$$
P_{0}=\left(\begin{array}{ccc}
0 & 0.5009 & 0.2700 \\
0.522 & 0 & 0.0050 \\
0.1227 & 0.3035 & 0
\end{array}\right), \quad P_{1}=\left(\begin{array}{ccc}
4.76 \times 10^{-6} & 0.0751 & 0.1540 \\
0.1014 & 0.1438 & 0.2278 \\
0.0960 & 0.0129 & 0.4648
\end{array}\right)
$$

with exponential rates $\lambda=(2.9102,57.3193,93.3259)$ possesses the autocorrelation function

$$
\rho(1)=-0.00028, \quad \rho(2)=0.0019, \quad \rho(3)=0.00021, \quad \rho(4)=3.54 \times 10^{-5},
$$

which does not satisfy either Remark 2 or the left-hand side condition of (3.3). These examples illustrate that it is possible to go beyond (3.3) to get more flexible dependence structures by increasing the number of states of the MAP. Therefore, it is of interest to consider the properties of the autocorrelation function (2.6) for general $m$-state MAPs for future research. 
However, because of the increasing number of parameters (six defining the $\mathrm{MAP}_{2}$ and 14 defining the $\mathrm{MAP}_{3}$ ), it becomes a tedious and difficult task. It would be desirable to study whether it is possible to adopt a matrix approach so that the extension of the presented results to higher-order MAPs is more straightforward. Unfortunately, this does not seem to be an easy question and, to the best of the authors' knowledge, is still unanswered. Work on these problems is underway.

\section{Acknowledgements}

Support from Junta de Andalucía (project number 2009/FQM-329), Comunidad de MadridUC3M (project number CCG08/UC3M/HUM-4467), and the Spanish Ministry of Education and Science (project numbers CSD2006-00032 (Consolider Ingenio Mathematica) and 2007/04438/001) is acknowledged by the authors. We also thank Professor M. F. Pita and Juan de Dios del Pino Corredera for helpful discussions. Part of this work was done while the second author was visiting the Centre de Recerca Matemàtica, Barcelona.

\section{References}

[1] Chakravarthy, S. R. (2001). The batch Markovian arrival process: a review and future work. In Advances in Probability and Stochastic Processes, eds A. Krishnamoorthy, N. Raju and V. Ramaswami, Notable Publications, NJ, pp. 21-49.

[2] Kang, S. H., Han Kim, Y., Sung, D. K. And Chol, B. D. (2002). An application of Markovian arrival process to modeling superposed ATM cell streams. IEEE Trans. Commun. 50, 633-642.

[3] Lucantoni, D. M. (1993). The BMAP/G/1 queue: a tutorial. In Performance Evaluation of Computer and Communication Systems (Lecture Notes Comput. Sci. 729), eds L. Donatiello and R. D. Nelson, Springer, New York, pp. 330-358.

[4] Montoro-Cazorla, D., Pérez-Ocón, R. and Segovia, M. (2009). Replacement policy in a system under shocks following a Markovian arrival process. Reliab. Eng. System Safety 94, 497-502.

[5] Neuts, M. F. (1979). A versatile Markovian point process. J. Appl. Prob. 16, 764-779.

[6] Ramaswami, V. (1980). The N/G/1 queue and its detailed analysis. Adv. Appl. Prob. 12, 222-261.

[7] Ramírez-Cobo, P., Lillo, R. And Wiper, M. (2010). Bayesian inference for the two-state Markovian arrival process. Working paper 10-43, Statistics and Econometrics Series 26, Universidad Carlos III de Madrid.

[8] Ramírez-Cobo, P., Lillo, R. E. ANd Wiper, M. P. (2010). Nonidentifiability of the two-state Markovian arrival process. J. Appl. Prob. 47, 630-649.

[9] RydÉn, T. (1996). An EM algorithm for estimation in Markov-modulated Poisson processes. Comput. Statist. Data Anal. 21, 431-447.

[10] ScotT, S. L. (1999). Bayesian analysis of the two state Markov modulated Poisson process. J. Comput. Graphical Statist. 8, 662-670. 